\title{
95. Elliptic Factors of Selberg Zeta Functions
}

\author{
By Masao TSUZUKI \\ Department of Mathematical Sciences, University of Tokyo \\ (Communicated by Shokichi IYANAGA, M. J. A., Dec. 13, 1993)
}

We show that the elliptic factors of Selberg zeta functions are expressed in terms of multiple gamma functions.

§1. Elliptic factors. Let $X=G / K$ be a rank one symmetric space of non compact type, where $G$ is a connected semisimple Lie group with finite center, and $K$ is a maximal compact subgroup of $G$. Put $g=\operatorname{Lie}(G)$, $\mathfrak{k}=\operatorname{Lie}(K)$ and let $\mathfrak{g}_{\boldsymbol{C}}, \mathfrak{l}_{\boldsymbol{C}}$ be their complexifications. We assume that $\operatorname{rank} G$ $=\operatorname{rank} K$ and fix a Cartan subgroup $T$ of $G$ which is contained in $K$. We choose a system of positive roots of $\Phi\left(\mathrm{g}_{\boldsymbol{C}}, \mathrm{t}_{\boldsymbol{C}}\right)$ and a singular imaginary root $\alpha_{t}$ in $\Phi^{+}\left(g_{C}, t_{C}\right)$. Let $G=K A_{R} N$ be an Iwasawa decomposition of $G$. From the assumption, $A_{R}$ is a one dimensional real torus. We identify $a_{R}$ with $\boldsymbol{R}$ as in [11]. Let $\rho_{0}$ be the half of the sum of positive roots in $\Phi\left(\mathfrak{g}, \mathfrak{a}_{R}\right)$. Let $M$ be the centralizer of $A_{R}$ in $K$, and $A_{I}$ be a Cartan subgroup of $M$. Let $\mathfrak{m}, \mathfrak{a}_{R}, \mathfrak{a}_{I}$ be the Lie algebras of $M, A_{R}, A_{I}$, and $\mathfrak{m}_{\boldsymbol{C}}, \mathfrak{a}_{R, C}, \mathfrak{a}_{I, C}$ their complexifications respectively. Let $\Gamma$ be a discrete subgroup of $G$ such that $\operatorname{vol}(G / \Gamma)<\infty$. We define the elliptic factor of the Selberg zeta function for $(G, \Gamma)$ as a smooth function $Z_{e l l}(s)$ on a half interval $(a, \infty)$ of $\boldsymbol{R}$ which satisfies the identity

$$
\left(-\frac{1}{2\left(s-\rho_{0}\right)} \frac{d}{d s}\right)^{m} \log Z_{e l l}(s)=\int_{0}^{+\infty} I_{e l l}\left(h_{t}\right) e^{-s\left(s-2 \rho_{0}\right) t} t^{m-1} d t
$$

for a positive integer $m$, where $I_{\text {ell }}$ denotes the elliptic term of the Selberg trace formula for $(G, \Gamma)$, and $h_{t}$ is the spherical fundamental solution of the heat equation $\left(\Delta+\frac{\partial}{\partial t}\right) u=0$ on $X$. By this definition $Z_{e l l}(s)$ is determined up to a factor $\exp \left(P\left(s-\rho_{0}\right)\right)$, where $P(s)$ is an even polynomial. We calculate the right hand side of $(*)$ using the Fourier inversion formula of elliptic orbital integrals [11], and determine $Z_{e l l}(s)$ as a finite product of multiple gamma functions.

§2. Results. Let $\mathscr{E}_{\Gamma}$ be the set of elliptic conjugacy classes of $\Gamma$, consisting of all conjugacy classes of finite orders. For $\gamma \in \mathscr{E}_{\Gamma}$, we denote by $n_{\gamma}$ its order. We choose an element $t_{\gamma}$ of $T$ which is conjugate to $\gamma$ in $G$; $t_{\gamma}$ is unique up to the action of the Weyl group $W=W(G, T)$. Let $G_{r}$ be the centralizer of $t_{\gamma}$ in $G$ and $g_{\gamma}$ be its Lie algebra. We write $\Phi_{\gamma}^{+}, \Phi_{I}^{+}$the sets of all positive roots in $\Phi\left(\mathfrak{g}_{r, C}, \mathrm{t}_{C}\right), \Phi_{I}=\Phi\left(\mathfrak{m}_{C}, \mathfrak{a}_{I, C}\right)$ and $r_{r}, r_{I}$ their cardinalities respectively. For each element $w \in W$, put

$$
P_{r, w}(\nu)=\prod_{\beta \in \Phi_{r}^{+}}\left(w\left(-\rho_{I}+\nu \alpha_{t}\right), \beta\right) \quad(\nu \in \boldsymbol{R}),
$$

where $\rho_{I}$ is the half of the sum of roots in $\Phi_{I}^{+}$. For an integral $\lambda \in \sqrt{-1} \mathrm{t}$, 
denote $\xi_{\lambda}$ the corresponding unitary character of $T$. We define complex numbers $\theta_{r}^{(j)}(\gamma)\left(1 \leqslant j \leqslant r_{r}\right)$ as follows :

where

$$
\theta_{r}^{(j)}(\gamma)=d(\gamma)^{-1}(-1)^{q_{\gamma}} \sum_{w \in W / W_{r}}(-1)^{l(w)} P_{\gamma, w}^{(j)}\left(\frac{r}{2}\right) \xi_{w\left(-\rho_{I}+\frac{\gamma}{2} \alpha_{t}\right)}\left(t_{\gamma}\right),
$$

$$
d(\gamma)=\left|W_{\gamma}^{C}\right|\left[G_{\gamma}: G_{\gamma}^{0}\right]\left(\prod_{\beta \in \Phi_{r}^{+}}\left(\rho_{\gamma}, \beta\right)\right) \xi_{\rho}\left(t_{\gamma}\right) \prod_{\beta \in \Phi^{+}-\Phi_{r}^{+}}\left(1-\xi_{-\beta}\left(t_{r}\right)\right),
$$

$r$ is an integer such that $-\rho_{I}+\frac{r}{2} \alpha_{t}$ is a $\Phi\left(g_{C}, t_{C}\right)$-integral element, $P_{r, w}^{(j)}$ means $j$-th derivative of $P_{r, w}, W_{r}=W\left(G_{r}^{0}, T\right), W_{r}^{C}=W\left(\mathrm{~g}_{r, \boldsymbol{C}}, \mathrm{t}_{\boldsymbol{C}}\right), q_{r}=\frac{1}{2}$ $\operatorname{dim}\left(G_{\gamma} / K_{r}\right), q=\frac{1}{2} \operatorname{dim}(G / K)$, and $\rho_{r}=\frac{1}{2} \sum_{\beta \in \Phi_{r}^{+}} \beta$. We take the Euler Poincaré measure on $G_{\gamma}$ as in [3]. We now state our main theorem.

Theorem. (1) There exists a smooth function $Z_{\text {ell }}(s)$ which satisfies (*), and it is expressed in $\operatorname{Re}(s)>\rho_{0}$ as follows:

where

$$
Z_{e l l}(s)=\prod_{r \in \mathbb{B}_{\Gamma}} Z_{r}(s)^{\operatorname{vol}\left(\Gamma_{r} \backslash G_{r}\right)}
$$

$$
\begin{aligned}
& Z_{r}(s)= \prod_{\substack{0 \leqslant r<2 n_{r} \\
r=\varepsilon(\bmod 2)}}\left(\prod_{j=0}^{r_{r}} G_{j+1}\left(\frac{s-\rho_{0}+r}{2 n_{r}}\right)^{c_{r}^{(j)}(r)}\right), \\
& c_{r}^{(j)}(\gamma)= \frac{(-1)^{r_{I}} n^{j}}{j !}\left(\theta_{-r}^{(j)}(\gamma)(-1)^{j+1}+\theta_{r}^{(j)}(\gamma)\right), \\
& \varepsilon= \begin{cases}0 & (G=S U(2 n, 1)) . \\
1 & (\text { otherwise })\end{cases}
\end{aligned}
$$

$G_{j}(z)$ is a multiple gamma function of Barnes, and defined by means of Weierstrass product as follows ([1], [8], [9]).

$$
G_{j+1}(s)^{-1}=\exp \left(\frac{(-1)^{j} \gamma}{j+1} s^{j+1}+\frac{(-1)^{j}}{j} s^{j}\right) \underset{n \geqslant 1}{\prod_{j+1}} P_{j}\left(\frac{-s}{n}\right)^{n^{j}},
$$

where $P_{j}(x)=(1-x) \exp \left(x+\frac{x^{2}}{2}+\cdots+\frac{x^{j}}{j}\right)$, and $\gamma$ is the Euler constant.

(2) When $\Gamma$ is cocompact, the above $Z_{\text {ell }}(s)$ has a meromorphic continuation to the whole complex plane and has possible simple poles or zeros at $s=\rho_{0}+l$ for $l \in \boldsymbol{Z}$ with $l \equiv \varepsilon \bmod 2$. The order of zero at $s=\rho_{0}+l$ is given by

$$
(-1)^{r_{I}} \sum_{\gamma \in \mathbb{B}_{\Gamma}} \operatorname{vol}\left(\Gamma_{\gamma} \backslash G_{\gamma}\right)\left(\theta_{-l}^{(0)}(\gamma)-\theta_{l}^{(0)}(\gamma)\right)
$$

Remarks. (1) An explicit form of $Z_{\gamma}(s)$ for central $\gamma$ 's have been obtained by Kurokawa by different method ([6], [7]).

(2) For cocompact $\Gamma$, the number

$$
\sum_{r \in \mathbb{B}_{\Gamma}} \operatorname{vol}\left(\Gamma_{\gamma} \backslash G_{\gamma}\right) \theta_{-l}^{(0)}(\gamma)
$$

appearing in the above formulae is, up to a sign, identified with an alternating sum of dimensions of certain $L^{2}$-cohomology spaces in [3].

(3) We define the completed Selberg zeta function $\hat{Z}(s)$ as follows:

$$
\hat{Z}(s)=Z_{i d}(s) Z_{e l l}(s) Z_{p a r}(s) Z_{\Gamma}(s)
$$


where the first three factors of the right hand side are regarded as gamma factors of the zeta function. According to the recent results of Jorgenson and Lang [4], one can obtain determinant expression of $\hat{Z}(s)$ under the assumption that its gamma factors are themselves expressed as regularized products. This assumption is satisfied for the identity factor and the elliptic factor by the results of Kurokawa [7] and ours. By using the results of Reznikov [10], the parabolic factor also has determinant expression when $G$ is $S O(2 n, 1)(n \geqslant 1)$ or $S U(2 n, 1)(n \geqslant 1)$ and $\Gamma$ is its congruence subgroup. The regularized products expression of $\hat{Z}(s)$ are known in some special cases [5].

§3. Proof of the theorem. By definition, we have

$$
I_{e l l}\left(h_{t}\right)=\sum_{r \in \mathscr{B}_{\Gamma}} \operatorname{vol}\left(\Gamma_{r} \backslash G_{r}\right) \mathscr{O}_{r}\left(h_{t}\right) \quad(t>0),
$$

where $\mathscr{O}_{\gamma}\left(h_{t}\right)=\int_{G_{r} \backslash G} h_{t}\left(x^{-1} t_{r} x\right) \frac{d x}{d x_{r}}$.

From the Fourier inversion formula of [11], $\mathscr{O}_{r}\left(h_{t}\right)$ can be written as a finite linear combination of integrals of following type :

$$
h_{\theta}^{\varepsilon}(P ; t)=\int_{-\infty}^{+\infty} 2 \hat{h}_{t}(\nu) \frac{e^{\nu \theta} P\left(\frac{\sqrt{-1} \nu}{2}\right)-(-1)^{\varepsilon} e^{-\nu \theta} P\left(-\frac{\sqrt{-1} \nu}{2}\right)}{e^{\pi \nu / 2}-(-1)^{\varepsilon} e^{-\pi \nu / 2}} d \nu,
$$

where $P$ is a polynomial with coefficients in $\boldsymbol{R}, \varepsilon \in\{0,1\}$, and $\theta$ is a real number in $\left[-\frac{\pi}{2}, \frac{\pi}{2}\right]$. Hence, the calculation of $Z_{e l l}(s)$ is reduced to that of the integrals

$$
\Psi_{r}(s, m)=\int_{0}^{\infty} e^{-s\left(s-2 \rho_{0}\right) t} \mathscr{O}_{r}\left(h_{t}\right) t^{m-1} d t
$$

and

$$
I_{\theta}^{\varepsilon}(P ; s, m)=\int_{0}^{\infty} e^{-t\left(s-\rho_{0}\right)^{2}} h_{\theta}^{\varepsilon}(P ; t) t^{m-1} d t
$$

This is given as follows. Let $\theta=\frac{l \pi}{n}(l, n \in \boldsymbol{Z},-\pi \leqslant 2 \theta \leqslant \pi)$, and $\varepsilon \in$ $\{0,1\}$. We first obtain

$$
\begin{aligned}
I_{\theta}^{\varepsilon}(P ; s, m) & =\left(-\frac{1}{2\left(s-\rho_{0}\right)} \frac{d}{d s}\right)^{m-1} I_{\theta}^{\varepsilon}(P ; s) \\
2\left(s-\rho_{0}\right) I_{\theta}^{\varepsilon}(P ; s)=\frac{\sqrt{-1}}{n} & \sum_{\substack{0 \leqslant r<4 n \\
r=\varepsilon(\bmod 2)}} \phi\left(\frac{s-\rho_{0}+r}{4 n}\right) \\
& \times\left\{P\left(\frac{s-\rho_{0}}{2}\right) e^{-\frac{\pi-2 \theta}{2} r \sqrt{-1}}-P\left(\frac{\rho_{0}-s}{2}\right) e^{\frac{\pi-2 \theta}{2} r \sqrt{-1}}\right\},
\end{aligned}
$$

where $\phi(s)$ is the logarithmic derivative of the gamma function, and $m>\operatorname{deg}(P)$. Let $\phi_{j+1}(s)=\frac{d}{d s} \log G_{j+1}(s)(s \gg 0)$, then $Q_{j}(s)=\phi_{j+1}(s)-$ $s_{j} \phi(s)$ is a polynomial. So it holds that

$$
P\left(\frac{\sigma}{2}\right) \phi\left(\frac{\sigma+i}{2 n}\right)=\sum_{j=0}^{\operatorname{deg}(P)} \frac{(-n)^{j}}{j !} P^{(j)}\left(\frac{-i}{2}\right) \phi_{j+1}\left(\frac{\sigma+i}{2 n}\right)+R(s)
$$


for a polynomial $R(s)$. Thus we have

$$
\begin{aligned}
2\left(s-\rho_{0}\right) I_{\theta}^{\varepsilon}(P ; s) & =\frac{\sqrt{-1}}{n} \sum_{\substack{0 \leqslant r<4 n \\
r=\varepsilon(\bmod 2)}} \sum_{j=0}^{\operatorname{deg}(P)} \frac{(-n)^{j}}{j !} \phi_{j+1}\left(\frac{s-\rho_{0}+r}{4 n}\right) \\
& \times\left\{P(j)\left(\frac{-i}{2}\right) e^{-\frac{\pi-2 \theta}{2} r \sqrt{-1}}-P^{(j)}\left(\frac{i}{2}\right) e^{\frac{\pi-2 \theta}{2} r \sqrt{-1}}\right\}+R(s)
\end{aligned}
$$

for a polynomial $R(s)$ and $m>\operatorname{deg}(P)$. Now from the results of [11], we have

$$
\begin{aligned}
\Psi_{r}(s, m)= & \frac{\sqrt{-1}\left|W_{\gamma}\right|(-1)^{r_{I}}}{8\left|W\left(M, A_{I}\right)\right| d(\gamma)} \sum_{u \in W_{I}}(-1)^{l(u)} \sum_{w_{i}}(-1)^{l\left(w_{i}\right)} \\
& \times \bar{\chi}_{I}^{u}\left(t_{\gamma}\right)\left(\operatorname{sgn} \theta_{i}\right)^{\varepsilon} I_{\theta_{i}+s g n \theta_{i} \frac{\pi}{2}}^{\varepsilon}\left(P_{r, w_{i} u} ; s, m\right),
\end{aligned}
$$

where $\chi_{I}$ is the unitary character of $A_{I}$ with differential $\rho_{I},\left\{w_{i}\right\}$ is a complete set of representative of $W_{r}$ in $W$, and for each $w_{i}, \theta_{i}$ is the real number such that $\xi_{\alpha_{t}}\left(w_{i}^{-1} t_{r}\right)=e^{-2 \sqrt{-1} \theta_{i}}\left(-\pi \leqslant 2 \theta_{i}<\pi\right)$, and $\varepsilon$ is given in the theorem. Consequently, we obtain $\Psi_{\gamma}(s, m)$ for $m>r_{r}$ as follows.

where

$$
\Psi_{r}(s, m)=\left(-\frac{1}{2\left(s-\rho_{0}\right)} \frac{d}{d s}\right)^{m-1} \Psi_{r}(s),
$$

$$
2\left(s-\rho_{0}\right) \Psi_{r}(s)=\sum_{\substack{0 \leq r<2 n_{r} \\ r=\varepsilon(\bmod 2)}} \sum_{j=0}^{r_{r}} c_{r}^{(j)} \frac{1}{2 n_{r}} \psi_{j+1}\left(\frac{s-\rho_{0}+r}{2 n_{r}}\right) .
$$

This gives our expression of $Z_{e l l}(s)$ via multiple gamma functions.

Since $\phi_{j+1}(s)$ has simple poles at $s=-n(n \in \boldsymbol{Z}, n \leqslant 0)$, with residue $-(-n)^{j}$,

$$
\Psi(s)=\sum_{r \in \mathbb{B} \Gamma} \operatorname{vol}\left(\Gamma_{r} \backslash G_{r}\right) \Psi_{r}(s)
$$

has a possible simple pole at $s \stackrel{r \in \mathscr{R} \Gamma}{=} \rho_{0}+l(l \in \boldsymbol{Z}, l \equiv \varepsilon(\bmod 2))$ and

$$
\operatorname{Res}_{s=\rho_{0}+l} \Psi(s)=(-1)^{r_{I}} \sum_{\gamma} \operatorname{vol}\left(\Gamma_{\gamma} \backslash G_{\gamma}\right)\left(\theta_{-l}^{(0)}(\gamma)-\theta_{l}^{(0)}(\gamma)\right) \text {. }
$$

When $G / \Gamma$ is compact, this is an integer [3]. Thus we have a meromorphic $Z_{e l l}(s)$ satisfying $\frac{d}{d s} \log Z_{e l l}(s)=\Psi(s)$.

\section{References}

[1] Barnes, E. W.: On the theory of the multiple gamma function. Trans. Cambridge Philos. Soc., 19, 375-425 (1904).

[2] Gangolli, R. : Zeta functions of Selberg's type for compact space forms of symmetric spaces of rank 1. Illinois Jour. Math., 21, 1-41 (1977).

[ 3 ] Hotta, R., and Parthasarathy., R.: Multiplicity formulae for discrete series. Inv. Math., 26, 133-178 (1974).

[ 4 ] Jorgension, J., and Lang, S.: On Cramers theorem for general Euler products with functional equation (1993) (preprint).

[ 5 ] Koyama, S.: Determinant expression of Selberg zeta functions. 1 ; 2 . Trans. Amer. Math. Soc. , 324,149-168 (1991); 329, 755-772 (1991).

[6] Kurokawa, N.: Multiple sine functions and Selberg zeta functions. Proc. Japan Acad., 67A, 61-64 (1991).

[ 7 ] - Gamma factors and Plancherel measures. ibid., 68A, 256-260 (1992). 
[ 8 ] Kurokawa, N.: Lectures on multiple sine functions (at Univ. of Tokyo) (1991).

[9] Manin, Y. I.: Lectures on zeta functions and motives. Max Planck Institute preprint (1992).

[10] Reznikov, A.: Eisenstein matrix and existence of cusp forms. G. A. F. A., 3, 79-105 (1993).

[11] Sally, P. J., and Warner. G.: The Fourier transform on semisimple Lie group of real rank one. Acta. Math., 131, 1-26 (1973).

[12] Vignéras, M. F.: L'equation fonctionelle de la fonction zéta de Selberg du groupe modulaire PSL (2,Z). Astérisque, 61, 235-249 (1979). 